NYU-TH-96/09/01 hep-th/9609073

\title{
Spontaneous Breaking of Extended Supersymmetry in Global and Local Theories *
}

\author{
M. Porrati ${ }^{\mathrm{a}} \dagger$ \\ a Department of Physics, NYU \\ 4 Washington Pl. New York, NY 10003, USA
}

We review the "no-go" theorems that severely constrain the breaking of $N=2$ supersymmetry to $N=1$ (both in rigid supersymmetry and supergravity), and we exhibit some models that evade them.

\section{A Theorem and its Loophole}

A well known theorem [1] ensures that extended rigid supersymmetry cannot be broken partially: either all supersymmetries are broken or all are exact. The heuristic proof of this theorem follows from the $N$-extended SUSY algebra:

$\left\{\bar{Q}_{\dot{\alpha}}^{i}, Q_{\alpha}^{j}\right\}=2 \sigma_{\dot{\alpha} \alpha}^{\mu} \delta^{i j} P_{\mu}, \quad i=1, . ., N$.

The Hamiltonian of a supersymmetric theory is manifestly positive, because, thanks to eq. (1), it can be expressed as

$H=\frac{1}{2} \sum_{\alpha} Q_{\alpha}^{\dagger i} Q_{\alpha}^{i}$, no sum over $i$.

If a supersymmetry, say $i=1$, is broken, then its generator does not annihilate the vacuum: $Q^{1}|0\rangle \neq 0$. Equation (2) then implies that $\langle 0|H| 0\rangle \neq 0$, i.e. that $Q^{i}|0\rangle \neq 0$ for all $i$. This argument can be made rigorous either by working with a theory in a finite periodic box (thereby giving up Lorentz invariance), or by using the SUSY current algebra

$$
\begin{aligned}
\lim _{V \rightarrow \infty}\left\{\bar{Q}_{V \dot{\alpha}}^{i}, J_{\mu \alpha}^{j}(x)\right\} & =2 \sigma_{\dot{\alpha} \alpha}^{\nu} \delta^{i j} T_{\mu \nu}(x), \\
\bar{Q}_{V \dot{\alpha}}^{i} & \equiv \int_{V} d^{3} \vec{y} \bar{J}_{0 \dot{\alpha}}^{i}(\vec{y}, t) .
\end{aligned}
$$

Here $T_{\mu \nu}$ and $J_{\mu \alpha}^{i}$ are the stress-energy tensor and the supercurrent, respectively. The infinite

\footnotetext{
*Contribution to the proceedings of the Spring School and Workshop on String Theory, Gauge Theory and Quantum Gravity, ICTP, Trieste, Italy, March 18-29, 1996.

†Supported in part by NSF under grant PHY-9318781
}

volume limit in this equation exists even when SUSY is broken?, because the (anti)-commutator of local (gauge-invariant) operators is always local.

The strength of SUSY breaking is parametrized by the matrix element of the SUSY current in between the vacuum and the (would-be) goldstino states

$$
\left\langle p \xi j\left|J_{\mu \alpha}^{i}(x)\right| 0\right\rangle=\bar{v}^{\dot{\alpha} j} \sigma_{\mu \dot{\alpha} \alpha} F_{j}^{i} e^{i p x} .
$$

By computing the vacuum expectation value of eq. (3), and using eq. (4), one finds that $F_{l}^{* i} F_{j}^{l} \propto$ $\delta_{j}^{i}\left\langle 0\left|T_{\mu}^{\mu}\right| 0\right\rangle$. The SUSY algebra has an $S U(N)$ symmetry that acts by unitary transformations on the index $i$, and that can be used to bring the goldstino-coupling matrix into the form $F_{j}^{i}=$ $F_{g} \delta_{j}^{i}$. This shows that either $F_{g}=0$, i.e. all supersymmetries are exact, or $F_{g} \neq 0$, that is they are all broken with equal strength.

This "no-go" theorem can be evaded, as often goes with no-goes, by changing its assumptions. They can be changed in two ways

1. If the Hilbert space of the theory is not positive definite, then $Q^{i}|0\rangle \neq 0$ can be consistent with $\left\langle 0\left|Q^{\dagger i} Q^{i}\right| 0\right\rangle=0$. This is what happens in covariant formulations of supergravity.

2. The SUSY current algebra is modified. This happens in supergravity when the

\footnotetext{
${ }^{3}$ That is when, rigorously speaking, the supercharge does not exist as an operator in the Hilbert space.
} 
local supersymmetry is gauge-fixed noncovariantly. More surprisingly, the SUSY current algebra may be modified even in rigid supersymmetry [2]:3].

In the latter case the modification reads

$$
\begin{aligned}
\lim _{V \rightarrow \infty}\left\{\bar{Q}_{V \dot{\alpha}}^{i}, J_{\mu \alpha}^{j}(x)\right\}= & 2 \sigma_{\dot{\alpha} \alpha}^{\nu} \delta^{i j} T_{\mu \nu}(x)+ \\
& +\sigma_{\mu \dot{\alpha} \alpha} C^{i j} .
\end{aligned}
$$

The additional term $C^{i j}$ is a constant, thus, the supersymmetry algebra on local operators is not modified by its presence. By studying the SUSY algebra on local operators only, one would never be able to detect the presence of $C^{i j}$, yet this term is enough to invalidate the "no-go" theorem!

The question remains as to whether such a constant nonzero term can arise in a local field theory with rigid $N=2$ supersymmetry.

The answer was given in ref. [4]. [1] explicitly construct an $N=2$ theory (hereafter called the APT model), with nonzero $C^{i j}$. The field content of the theory is a single $N=2$ vector multiplet, whose helicity content is $[(1) \pm 1,(2) \pm 1 / 2,(2) 0]$.

The $N=2$ Lagrangian of the vector multiplet is completely determined by an analytic function (the prepotential) $\mathcal{F}(z)$ ( $z$ is the complex scalar in the vector multiplet), and by two constant vectors of the $S U(2)$ isomorphism group of the $N=2$ superalgebra, $\vec{E}, \vec{M}[\mathbb{A}$. $\vec{E}$ is the standard (electric) $N=2$ Fayet-Iliopoulos term, while the magnetic F-I term $\vec{M}$ was introduced first in $[4]$. With an $S U(2)$ rotation they can be cast in the form

$\operatorname{Re} \vec{E}=\Lambda^{2}(0, e, \xi), \quad \vec{M}=\Lambda^{2}(0, m, 0)$.

The kinetic term of all fields in the multiplet is given by $\tau_{2}(z)=\operatorname{Im} \mathcal{F}^{\prime \prime}(z)>0$, while the theta angle is $\tau_{1}(z)=\operatorname{Re} \mathcal{F}^{\prime \prime}(z)$.

To find whether the $N=2$ supersymmetry is broken to $N=1$ one must look for Lorentzinvariant backgrounds on which the SUSY transformation laws of some field have a nonzero VEV. Lorentz invariance constrains the form of a nonzero VEV to

$\delta \lambda_{i}=\frac{i}{\sqrt{2}} Y^{A} \epsilon_{i j} \sigma_{k}^{A j} \eta^{k}$.

Here, $\lambda_{i}$ is the gaugino field (doublet of $S U(2)$ ), $\eta^{i}$ is the $N=2$ SUSY transformation parameter, and $Y^{A}, A=1,2,3$, is a yet unspecified (complex) vector of $S U(2)$. In ref. [1 it was shown that

$Y^{A}=-\frac{2}{\tau_{2}(z)}\left[\operatorname{Re} E^{A}+\tau_{1}(z) M^{A}\right]+2 i M^{A}$.

Partial breaking of $N=2$ to $N=1$ occurs if the matrix $Y^{A}(a) \epsilon_{i j} \sigma_{k}^{A j}$ has a non-degenerate zero eigenvalue. This happens at

$\tau_{1}(z)=-e / m, \tau_{2}(z)=m / \xi$.

Generically, these equations can be solved for $z$ if $m / \xi>0$, and $\mathcal{F}^{\prime \prime}(a)$ is not a constant, i.e. when the model is non-renormalizable and the magnetic F-I term $\vec{M}$ is nonzero. A VEV $z^{o}$ that solves eq. (9) is also a stable minimum of the scalar potential. This is a general result, valid whenever some supersymmetry is unbroken. It follows from the current algebra eq. (5), which implies the following identity for the scalar potential

$\left(\delta^{i} \lambda^{I}\right)^{*} Z_{I}^{J}(\phi) \delta^{j} \lambda_{J}=\delta^{i j} V(\phi)+C^{i j}$.

Here $\delta^{i} \lambda_{I}$ is the $i$-th SUSY transformation law of the spin- $1 / 2$ fields $\lambda_{I}$ (on a Lorentz-invariant background). The fermion kinetic term is $Z_{I}^{J}(\phi)$, $\phi$ denotes all scalar fields; $V(\phi)$ is the potential. If a supersymmetry, say $i=1$, is unbroken at $\phi=\phi^{o}$, then $\delta^{1} \lambda_{I}=0 \forall I$, and the component 11 of the matrix equation above implies

$V\left(\phi^{o}\right)=-C^{11},\left.\quad \frac{\partial V}{\partial \phi}\right|_{\phi^{o}}=0,\left.\quad \frac{\partial^{2} V}{\partial \phi^{2}}\right|_{\phi^{o}} \geq 0$.

Explicitly computing the current algebra of the APT model one finds that the field-independent term $C^{i j}$ is [3]

$C^{i j}=4 \epsilon_{A B C} \sigma^{A i j} \operatorname{Re} E^{B} M^{C}$.

As expected, partial SUSY breaking in the APT model is possible only when $C^{i j} \neq 0$.

Since the vacua given in eq. (9) are $N=1$ supersymmetric, the $N=2$ vector multiplet decomposes into $N=1$ multiplets, specifically, into a massless vector multiplet and a massive chiral multiplet.

\section{Supergravity and Partial Breaking}

In supergravity theories, there is no simple general argument forbidding partial breaking, not 
even at zero cosmological constant. Some constraints nevertheless exist.

To be concrete, let us examine the case of $N=$ 2 supergravity. First of all, the minimal field content of an $N=2$ theory that may allow for partial breaking to $N=1$ is, besides the $N=2$ gravitational multiplet, a vector multiplet and a hypermultiplet [5]. The helicity content of the gravitational multiplet is $[(1) \pm 2,(2) \pm 3 / 2,(1) \pm 1]$; the content of the hypermultiplet is $[(2) \pm 1 / 2,(4) 0]$. The vector multiplet is necessary because partial breaking to $N=1$ requires that one of the spin$3 / 2$ fields becomes massive. A massive spin-3/2 multiplet of $N=1$ contains two massive vectors, while the pure gravitational multiplet of $N=2$ contains only one such field. Since the scalars in the vector multiplet are neutral under the $U(1)$ 's gauged by the spin- 1 fields, a (charged) hypermultiplet is needed to give mass to the vectors by the Higgs effect.

A more technical constraint follows from the details of the construction of $N=2$ supergravity.

In an $N=2$ supergravity, the scalars in the vector multiplet, $z^{a}, a=1, . ., n_{V}$, parametrize a "special Kähler" manifold, i.e. a Kähler manifold endowed with a symplectic bundle of dimension $2 n_{V}+2$ which possesses a holomorphic section $Z^{\alpha}(z), \alpha=1, . ., 2 n_{V}+2$. The Kähler potential is completely determined by the section as [6]

$K=-\log i \bar{Z}^{\alpha}(z) \omega_{\alpha \beta} Z^{\beta}(z)$,

where $\omega_{\alpha \beta}$ is the (constant) symplectic norm of the bundle. Symplectic transformations on the bundle also act as electric-magnetic dualities on the $n_{V}+1$ vector fields ( the matter vectors plus the graviphoton).

The hypermultiplets, on the other hand, live in a quaternionic manifold [7]. They can be charged under the $n_{V}+1$ vectors. When these vectors are Abelian, the most general charge allowed by the DSZ quantization conditions [8] is a $2 n_{V}+2-$ dimensional vector $q_{I}^{\alpha}$ obeying $q_{I}^{\alpha} \omega_{\alpha \beta} q_{J}^{\beta} \in Z$, with $I$ labelling the different hypermultiplets. To be able to describe the interactions of the hypermultiplets with a local Lagrangian, the charges must obey a stronger condition:

$q_{I}^{\alpha} \omega_{\alpha \beta} q_{J}^{\beta}=0, \quad \forall I, J$.

Obviously, there are at most $n_{V}+1$ linearly in- dependent $q_{I}^{\alpha}$ obeying such condition. Whenever eq. (14) holds, one can choose $n_{V}+1$ linearly independent vectors $c^{A \alpha}, A=0, . ., n_{V}$, obeying $q_{I}^{\alpha} \omega_{\alpha \beta} c^{A \beta}=0$. These vectors select a particular symplectic basis with coordinates

$X^{A}=c^{A \alpha} Z^{\alpha}, \quad F_{A}=c^{A \alpha} \omega_{\alpha \beta} Z^{\beta}$.

Two very different situations occur.

1. The $X^{A}$ are a good set of homogenous coordinates, i.e. the change of coordinates $z^{a} \rightarrow t^{a} \equiv X^{a} / X^{0}$ is invertible.

2. The change of coordinates $z^{a} \rightarrow t^{a}$ is not invertible.

In the first case, one can show that the $F_{A}$ are the gradient of a homogeneous function of degree two: $F_{A}=\partial F(X) / \partial X^{A}$, also called prepotential. This is the case studied in [9] using the $N=2$ superconformal tensor calculus. A theorem proved in [10] shows that in this case $N=2$ supersymmetry cannot break to $N=1$ with zero cosmological constant.

In the second case, the prepotential does not exist. Needless to say, this statement depends crucially on our choice of basis eq. (15). This choice is necessary to obtain a local $N=2$ Lagrangian.

This is also the case in which one can find a model with partial supersymmetry breaking in flat space. This model was first found in ref. 11 as a singular limit of a model constructed within the superconformal framework. In ref. [12] it was realized that the model is an example of $N=2$ supergravity without prepotential.

Specifically, the field content of the model is the minimal one necessary to break $N=$ 2 to $N=1$. The hypermultiplet scalars, $b^{0}, b^{1}, b^{2}, b^{3}$, parametrize the quaternionic manifold $S O(4,1) / S O(4)$. The two $U(1)$ symmetries, gauged by the graviphoton and by the matter photon, act as translations on the $b$ 's:

$D_{\mu} b^{u}=\partial_{\mu} b^{u}+i g \delta^{u 1} A_{\mu}^{g p h}+i \delta^{u 2} g^{\prime} A_{\mu}^{m a t}$.

The scalar of the vector multiplet parametrizes the coset manifold $S U(1,1) / U(1)$. The Kähler potential is standard: $K=-\log (z+\bar{z})$, but the symplectic basis is not:

$X^{0}=-\frac{1}{2}, X^{1}=\frac{i}{2}, F_{0}=i z, F_{1}=z$. 
Obviously, the transformation $z \rightarrow X^{0} / X^{1}=i$ is non-invertible.

An explicit computation [12] shows that the SUSY transformations of all fermions (i.e. the gaugini $\lambda_{i}$, the two gravitini $\psi_{\mu}^{i}$, and the two "hyperini" $\left.\zeta^{i}\right)$ are proportional to the same matrix:

$\delta$ fermions $\propto\left(\begin{array}{ll}g-g^{\prime} & 0 \\ 0 & g+g^{\prime}\end{array}\right)\left(\begin{array}{l}\eta_{1} \\ \eta_{2}\end{array}\right)$.

Partial breaking to $N=1$ occurs when $g= \pm g^{\prime}$.

The model has a flat scalar potential $V(z, \bar{z}, b)=0$. This is not a generic property: it is due to our choice of the Kähler manifold $S U(1,1) / U(1)$. The fields of the $N=2$ theory rearrange into $N=1$ multiplets. Specifically, into the gravitational multiplet of $N=1$, a massive spin-3/2 multiplet, and two chiral multiplets, which are massless due to the degeneracy of the potential. Generalizations of this model have been given in ref. |13

\section{The APT Model as a Flat Limit of $N=2$ Supergravity}

A natural question arising at this point is whether the APT model can be obtained from an $N=2$ supergravity in the limit of infinite Planck mass: $M_{P l} \rightarrow \infty$.

This is indeed the case, and the nature of the limit can be understood quite simply, even before a detailed analysis of specific models.

First of all, the masses of both $N=2$ gravitini should go to zero in the flat limit $M_{P l} \rightarrow \infty$. Indeed, a non-zero $m_{3 / 2}$ would produce in the flat limit a soft explicit breaking of supersymmetry.

Moreover, the limit should produce a non-zero $C^{i j}$ in the SUSY current algebra, or, equivalently, in eq. (10). The analog of eq. (10) in supergravity is the so-called T-identity 10,14.

$$
\begin{aligned}
& \left(\delta^{i} \lambda^{I}\right)^{*} Z_{I}^{J}(\phi) \delta^{j} \lambda_{J}-3 M_{P l}^{2} M^{* i l} M^{l j}= \\
& =\delta^{i j} V(\phi),
\end{aligned}
$$

where $M^{i j}$ is the gravitino mass matrix. Eq. (10) is recovered in the limit $M_{P l} \rightarrow \infty$ when $M^{i j} \rightarrow$ 0 , but $M_{P l} M^{i j} \rightarrow$ nonzero constant.

Having understood the scaling properties needed to recover the APT model, it is relatively easy to find an explicit supergravity with the right flat limit. The field content is as in Section 2, and the hypermultiplet scalars still parametrize the manifold $S O(4,1) / S O(4)$. Their coupling to the graviphoton and the matter photon is specified by the covariant derivative [3]

$$
\begin{aligned}
D_{\mu} b^{u}= & \partial_{\mu} b^{u}+i\left(g_{1} \delta^{u 3}+g_{2} \delta^{u 2}\right) A_{\mu}^{g p h}+ \\
& i g_{3} \delta^{u 2} A_{\mu}^{m a t} .
\end{aligned}
$$

The geometry of the vector multiplet is specified by a choice of symplectic section ${ }^{4}[3]$ :

$$
\begin{aligned}
& X^{0}=\frac{1}{\sqrt{2}}, \quad X^{1}=\frac{i}{\sqrt{2}} f^{\prime}(z), \\
& F_{0}=-\frac{i}{\sqrt{2}}\left[2 f(z)-z f^{\prime}(z)\right], \quad F_{1}=\frac{z}{\sqrt{2}},
\end{aligned}
$$

where $f(z)$ is an arbitrary analytic function, regular around $z=0$.

To recover the APT model in the limit $M_{P l} \rightarrow$ $\infty$, we must scale appropriately the couplings $g_{1}, g_{2}, g_{3}$ and the function $f(z)$. The SUSY parameter $\eta_{i}$ must also be rescaled according to its canonical dimensions, and the fermion kinetic term must be brought in canonical form. In 3 it was shown that the appropriate rescaling reads:

$$
\begin{aligned}
g_{1}= & \frac{\Lambda^{2}}{M_{P l}^{2}} \xi, \quad g_{2}=\frac{\Lambda^{2}}{M_{P l}^{2}} e, \\
g_{3}= & 2 \frac{\Lambda}{M_{P l}} m, \\
f(z)= & \frac{1}{2}+\frac{\Lambda}{M_{P l}} z+\frac{\Lambda^{2}}{M_{P l}^{2}} \phi(z)+ \\
& +\mathcal{O}\left(\Lambda^{3} / M_{P l}^{3}\right), \\
\lambda_{i} \rightarrow & \left(M_{P l} \Lambda^{2}\right)^{-1 / 2} \lambda_{i}, \zeta^{i} \rightarrow M_{P l}^{-3 / 2} \zeta^{i}, \\
\psi_{i \mu} \rightarrow & M_{P l}^{-3 / 2} \psi_{i \mu}, \quad \eta_{i} \rightarrow M_{P l}^{1 / 2} \eta_{i} .
\end{aligned}
$$

The limit is taken keeping the scale $\Lambda$, the dimensionless parameters $\xi, e, m$, and the function $\phi(z)$ constant. In this limit the entire gravitational multiplet, as well as the hypermultiplet, decouple from the vector multiplet. The gravitational multiplet and the hypermultiplet become a hidden sector, whose interactions with the vector multiplet (the "observable sector") are suppressed by inverse powers of $M_{P l}$. For instance, the kinetic term of the hypermultiplet scalars reads

\footnotetext{
4 This section is obtained by performing the symplectic transformation $X^{1} \rightarrow-F_{1}, F_{1} \rightarrow X^{1}$ on a basis with prepotential $F\left(X^{0}, X^{1}\right)=-i\left(X^{0}\right)^{2} f\left(X^{1} / X^{0}\right)$.
} 
$M_{P l}^{2} 2^{-1} b_{0}^{-2} \partial_{\mu} b^{u} \partial^{\mu} b^{u}$. In the limit $M_{P l} \rightarrow \infty$, the $b^{u}$ 's do not fluctuate and appear as coupling constants in the low-energy action 5 . By defining $\mathcal{F}(z)=z^{2}-2 i \phi(z)$, one finds that, in the flat limit, the observable sector of our model becomes identical with the APT model, with parameters given in eq. (6). In particular, using the same definition of $\tau(z)$ as in Section 1, the SUSY transformation laws of the gaugini on a Lorentz-invariant background read:

$$
\begin{aligned}
\delta \lambda_{i} & =X_{i j} \eta^{j}+\mathcal{O}\left(\Lambda^{3} / M_{P l}\right) \\
X_{i j} & =\sqrt{2} \frac{\Lambda^{2}}{b^{0} \tau_{2}(z)}\left\{\xi \sigma_{i j}^{1}+i[e+m \tau(z)] \delta_{i j}\right\} \eta^{j} .
\end{aligned}
$$

At $\tau_{1}(z)=-e / m, \tau_{2}(z)=\xi / m$, the matrix $X_{i j}$ has a nondegenerate zero eigenvalue. As expected from the general considerations outlined before, there the scalar potential has a stable minimum in $z$. At the minimum, the potential reads

$\left.V\right|_{\text {min }}=-\frac{\Lambda^{4}}{2\left(b^{0}\right)^{2}}\left[(2 m-\xi)^{2}+e^{2}\right]$

\section{Conclusions}

We have seen that global extended supersymmetry can indeed undergo partial spontaneous breaking, and we showed that what makes it possible, is a field-independent modification of the SUSY current algebra, which does not modifies the SUSY algebra on local, gauge-invariant operators. We have also shown that partial breaking of supersymmetry at zero cosmological constant can occur in extended supergravity, by studying the $N=2$ case in some details.

Finally, we have shown that the APT model of global extended supersymmetry with partial SUSY breaking can be obtained as a flat limit of a supergravity model. In our example, the supergravity model contains extra fields that decouple only in the flat limit. Supersymmetry is broken to $N=1$ in the observable (vector-multiplet) sector. As shown in [3], the hidden sector generically breaks $N=2$ to $N=0$, in which case the scalar potential has no stationary point in $b^{0}$ for

\footnotetext{
${ }^{5}$ This is what happens in string theory with the $S$ modulus.
}

large but finite $M_{P l}$. For the special choice of parameters $e=0, \xi=2 m$, the hidden sector too preserves $N=1$ supersymmetry, and the scalar potential has a flat direction in $b^{0}$, as it can be verified by inspection of eq. (24).

\section{REFERENCES}

1. E. Witten, Nucl.Phys. B188 (1981) 513.

2. J. Hughes, J. Liu and J. Polchinski, Phys. Lett. B180 (1986) 370.

3. S. Ferrara, L. Girardello and M. Porrati, Phys. Lett. B376 (1996) 275.

4. I. Antoniadis, H. Partouche and T.R. Taylor, Phys. Lett. B372 (1996) 83, hep-th/9512006.

5. S. Ferrara and P. van Nieuwenhuizen, Phys. Lett. B127 (1983) 70.

6. A. Ceresole, R. D' Auria, S. Ferrara and A. Van Proeyen, Nucl. Phys. B444 (1995) 92.

7. J. Bagger and E. Witten, Nucl. Phys. B222 (1985) 1.

8. P.A.M. Dirac, Proc. R. Soc. A133 (1931) 60; J. Schwinger, Phys. Rev. 144 (1966) 1087; 173 (1968) 1536; D. Zwanziger, Phys. Rev. 176 (1968) 1480; 1489.

9. B. de Wit, P.G. Lauwers and A. Van Proeyen, Nucl. Phys. B255 (1985) 569.

10. S. Cecotti, L. Girardello and M. Porrati, Phys. Lett. B145 (1984) 61; Nucl. Phys. B268 (1986) 265.

11. S. Cecotti, L. Girardello and M. Porrati, Phys. Lett. B168 (1986) 83.

12. S. Ferrara, L. Girardello and M. Porrati, Phys. Lett. B366 (1996) 155.

13. P. Fré, L. Girardello, I. Pesando and M. Trigiante, hep-th/9607032.

14. S. Cecotti, L. Girardello and M. Porrati, Proceedings of the IX Johns Hopkins Workshop (World Scientific, Singapore, 1985); S. Ferrara and L. Maiani, Proceedings of the $V$ SILARG Symposium (World Scientific, Singapore, 1986). 